\title{
A 10-s sprint performed prior to moderate-intensity exercise prevents early post-exercise fall in glycaemia in individuals with type 1 diabetes
}

\author{
V. A. Bussau • L. D. Ferreira - T. W. Jones • \\ P. A. Fournier
}

Received: 5 April 2007 / Accepted: 23 April 2007 / Published online: 22 June 2007

(C) Springer-Verlag 2007

\begin{abstract}
Aims/hypothesis We investigated whether a 10-s maximal sprint effort performed immediately prior to moderateintensity exercise provides another means to counter the rapid fall in glycaemia associated with moderate-intensity exercise in individuals with type 1 diabetes.

Materials and methods Seven complication-free type 1 diabetic males $(21.6 \pm 3.6$ years; mean $\pm \mathrm{SD})$ with $\mathrm{HbA}_{1 \mathrm{c}}$ levels of $7.4 \pm 0.7 \%$ injected their normal morning insulin dose and ate their usual breakfast. When post-meal glycaemia fell to $\sim 11 \mathrm{mmol} / \mathrm{l}$, participants were asked to perform a 10-s all-out sprint (sprint trial) or to rest (control trial) immediately before cycling at $40 \%$ of peak rate of oxygen consumption for $20 \mathrm{~min}$, with both trials conducted in a random counterbalanced order.

Results Sprinting did not affect the rapid fall in glycaemia during the subsequent bout of moderate-intensity exercise $(2.9 \pm 0.4 \mathrm{mmol} / 1$ in $20 \mathrm{~min} ; p=0.00$; mean $\pm \mathrm{SE})$. However, during the following $45 \mathrm{~min}$ of recovery, glycaemia in the control trial decreased by $1.23 \pm 0.60 \mathrm{mmol} / \mathrm{l} \quad(p=0.04)$
\end{abstract}

V. A. Bussau • L. D. Ferreira P. A. Fournier $(\bowtie)$

School of Human Movement and Exercise Science,

The University of Western Australia,

35 Stirling Highway,

Crawley, Perth, WA 6009, Australia

e-mail: fournier@cyllene.uwa.edu.au

T. W. Jones

Department of Endocrinology and Diabetes,

Princess Margaret Hospital,

Subiaco, WA, Australia

T. W. Jones

Centre for Child Health Research, Telethon Institute of Child

Health Research, The University of Western Australia,

Perth, WA, Australia while remaining stable in the sprint trial, subsequently decreasing in this latter trial at a rate similar to that in the control trial. The large increase in noradrenaline (norepinephrine) $(p=0.005)$ and lactate levels $(p=0.0005)$ may have contributed to the early post-exercise stabilisation of glycaemia in the sprint trial. During recovery, adrenaline (epinephrine) and NEFA levels increased marginally in the sprint trial, but other counter-regulatory hormones did not change significantly $(p<0.05)$.

Conclusions/interpretation A 10-s sprint performed immediately prior to moderate-intensity exercise prevents glycaemia from falling during early recovery from moderateintensity exercise in individuals with type 1 diabetes.

Keywords Exercise - Glycaemia - Glycemia .

Hypoglycaemia $\cdot$ Hypoglycemia $\cdot$ Physical activity

Recovery $\cdot$ Sprint $\cdot$ Type 1 diabetes

\author{
Abbreviations \\ CON control trial \\ SP sprint trial \\ $V \mathrm{O}_{\text {2peak }}$ peak rate of oxygen consumption
}

\section{Introduction}

Prolonged exercise of high intensity $(>10 \mathrm{~min}$ at $>80 \%$ peak rate of oxygen consumption; $\left.V \mathrm{O}_{2 \text { peak }}\right)$ differs from moderate-intensity exercise in that it causes an increase rather than a fall in glycaemia in insulin-treated individuals with type 1 diabetes [1]. For this reason, we recently examined whether the performance of one or several short maximal sprint efforts could offer an alternative means to counter the fall in glycaemia associated with moderate- 
intensity exercise or elevated plasma insulin levels in individuals with type 1 diabetes [2-4]. Surprisingly, we found that immediately after a bout of moderate-intensity exercise it is preferable to sprint for $10 \mathrm{~s}$ than to only rest as a means of countering a decrease in glycaemia during recovery [2]. Also, we showed that the fall in glycaemia during and after exercise of moderate intensity is not as marked if this type of exercise is interspersed with several $4 \mathrm{~s}$ sprints, despite more work being performed [4]. It is important to note that the sprints in these studies [2-4] were short enough $(4-10 \mathrm{~s})$ to be well tolerated by all participants.

Given the benefits of sprinting during or after moderateintensity exercise $[2,4]$, this raises the intriguing question of whether performing a short sprint effort immediately prior to, rather than after, moderate-intensity exercise may offer a novel way of preventing the rapid fall in glycaemia normally observed during and after moderate-intensity exercise. In other words, this study examines whether short-duration sprinting should be an integral part of the preparatory routine of individuals with type 1 diabetes before they engage in sustained physical activities of moderate intensity.

\section{Materials and methods}

Participants Seven males with type 1 diabetes, (age 21.6 \pm 3.6 years; BMI $26.7 \pm 4.3 \mathrm{~kg} / \mathrm{m}^{2} ; V \mathrm{O}_{2 \text { peak }} 45.2 \pm 5.0 \mathrm{ml} \mathrm{kg}{ }^{-1}$ $\min ^{-1}$; diabetes duration 9.5 \pm 3.3 years; $\mathrm{HbA}_{1 \mathrm{c}} 7.4 \pm 0.7 \%$; total daily insulin dose $94 \pm 38 \mathrm{U}$; all means \pm SD), who were free from diabetic complications, had undetectable levels of C-peptide and were hypoglycaemia-aware, gave informed consent in accordance with both the University of Western Australia and Princess Margaret Hospital Ethics Committees. Participants were not taking any prescribed medication other than insulin and had not changed their insulin regimen for at least 3 months prior to the study. Following a familiarisation session, during which anthropometric measurements were taken, $V \mathrm{O}_{2 \text { peak }}$ was determined as described previously [5]. The next two visits were the sprint (SP) and control rest (CON) trials conducted in a random counterbalanced order.

Experimental trials and assays $\mathrm{In}$ the $48 \mathrm{~h}$ prior to the experimental trial, participants were not allowed to exercise as antecedent exercise could have affected the endocrine response to exercise [6]. In addition, testing was rescheduled if participants had experienced a hypoglycaemic episode during the $48 \mathrm{~h}$ pre-trial phase. Following their arrival in the laboratory at 08:00 hours, participants self-administered their usual dose of morning insulin into their abdomen, with insulin dosage kept identical between trials (mean dose $15 \pm 2 \mathrm{U}$ short-acting insulin, $16 \pm 9 \mathrm{U}$ intermediate-acting insulin), and a catheter was inserted in the antecubital fossa. Blood glucose levels were similar $(p=0.843)$ prior to insulin injection, with levels of $10.1 \pm$ 1.4 and $10.3 \pm 1.5 \mathrm{mmol} / 1$ in the $\mathrm{SP}$ and $\mathrm{CON}$ trials, respectively. Participants then consumed breakfast to increase glycaemia above $11 \mathrm{mmol} / \mathrm{l}$. Breakfast food choice and nutritional content reflected that normally eaten by the participant, with breakfast kept the same $(p>0.05)$ for both trials (total energy $1,736 \pm 193 \mathrm{~kJ}$; relative energy content $56 \pm 3 \%$ carbohydrate, $17 \pm 1 \%$ protein, $27 \pm 4 \%$ fat). However, one of the control participants consumed additional carbohydrate to achieve post-breakfast glycaemia above $11 \mathrm{mmol} / \mathrm{l}$.

After breakfast, no physical activity was allowed and glycaemia peaked at $13.8 \pm 0.8$ and $13.9 \pm 0.50 \mathrm{mmol} / 1$ in the SP and CON trials, respectively $(p=0.890)$. When glycaemia post-breakfast fell back to $\sim 11 \mathrm{mmol} / \mathrm{l}$ (approximately $111 \pm 10 \mathrm{~min}$ SP and $106 \pm 8 \mathrm{~min} \mathrm{CON}$ after insulin injection; $p=0.564$ ), participants either rested or performed a 10-s maximal sprint effort on an airbraked cycle ergometer (Repco, Sydney, NSW, Australia). This was immediately followed by $20 \mathrm{~min}$ of moderateintensity exercise $\left(40 \% \quad V \mathrm{O}_{2 \text { peak }}\right)$, with the rationale underlying the intensity and duration of this bout of moderate-intensity exercise similar to that discussed previously [2]. Blood sampling and assays of metabolites and hormones were also performed as described in our earlier studies $[2,4]$.

Statistical analyses Data were analysed using a two-way repeated-measures ANOVA and Fisher's least significant differences test for a posteriori analysis with SPSS 13.0 for Windows software (SPSS, Chicago, IL, USA). Statistical significance was accepted at $p<0.05$. Participants' characteristics are expressed as means $\pm \mathrm{SD}$; all other results are means \pm SEM.

\section{Results and discussion}

Here we show that although a 10 -s sprint initiated immediately before moderate-intensity exercise did not affect the rapid decline in glycaemia during a subsequent bout of moderate-intensity exercise performed when plasma insulin levels are elevated, it did prevent the fall in glycaemia for at least the first $45 \mathrm{~min}$ of recovery (Fig. 1). This suggests that including a short sprint as part of the preparatory routine of individuals with type 1 diabetes before they engage in sustained moderate-intensity exercise might provide another means of temporarily stabilising glycaemia during early recovery. 


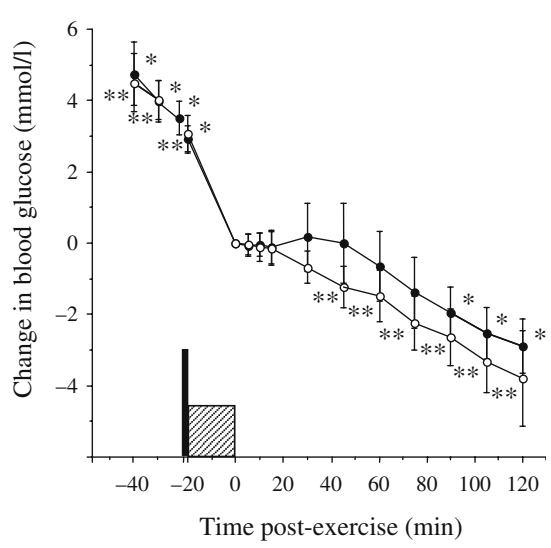

Fig. 1 Effect of a 10-s sprint on blood glucose levels during and after moderate-intensity exercise. The moderate-intensity exercise was commenced at time-point minus 20. Blood glucose levels are expressed relative to those immediately after moderate-intensity exercise (time-point $=$ zero). All values are shown as mean \pm SEM. Black box, sprint; hatched box, moderate-intensity exercise; closed circles, SP trial; open circles, CON trial. $* p<0.05$ for difference from 0 min post-moderate-intensity exercise time point in SP; $* *<0.05$ for difference from $0 \mathrm{~min}$ post-moderate-intensity exercise time point in $\mathrm{CON}$

The marked rise in noradrenaline (norepinephrine) and lactate levels immediately after exercise in the SP group might explain, in part, how sprinting prior to moderateintensity exercise delays the fall in glycaemia early into recovery. Noradrenaline levels during early recovery were elevated in the SP group while blood glucose levels were stable, but soon after the return of noradrenaline to basal preexercise levels, blood glucose levels in the SP and CON groups fell at comparable rates (Fig. 2). Although high catecholamine levels have been generally acknowledged to counter insulin-mediated falls in glycaemia [1], their role as counter-regulatory hormones during exercise in type 1 diabetic individuals is still controversial [7]. Elevated lactate levels (Fig. 2) are another factor that may have contributed to the stabilisation of glycaemia early in recovery by providing gluconeogenic precursors for hepatic glucose production and by increasing peripheral insulin resistance [8].

The patterns of change in the levels of insulin, cortisol, glucagon and growth hormones in the SP and CON groups suggest that these hormones are unlikely to explain the delayed fall in glycaemia during recovery in the SP group. Insulin and cortisol levels in SP and CON groups did not change significantly during recovery (Fig. 2). Also, the levels of glucagon did not increase significantly during and after exercise in the SP group. The question of whether portal glucagon levels responded in a similar manner remains to be established (Fig. 2). Finally, growth hormones are also unlikely to play a role as no significant changes in growth hormone levels were seen in either of the groups (Fig. 2). However, a close inspection of Fig. 2e suggests that significant changes in growth hormone levels a

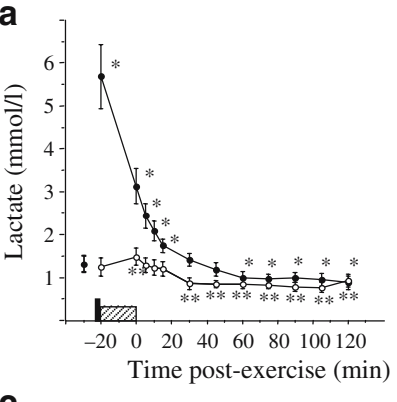

C
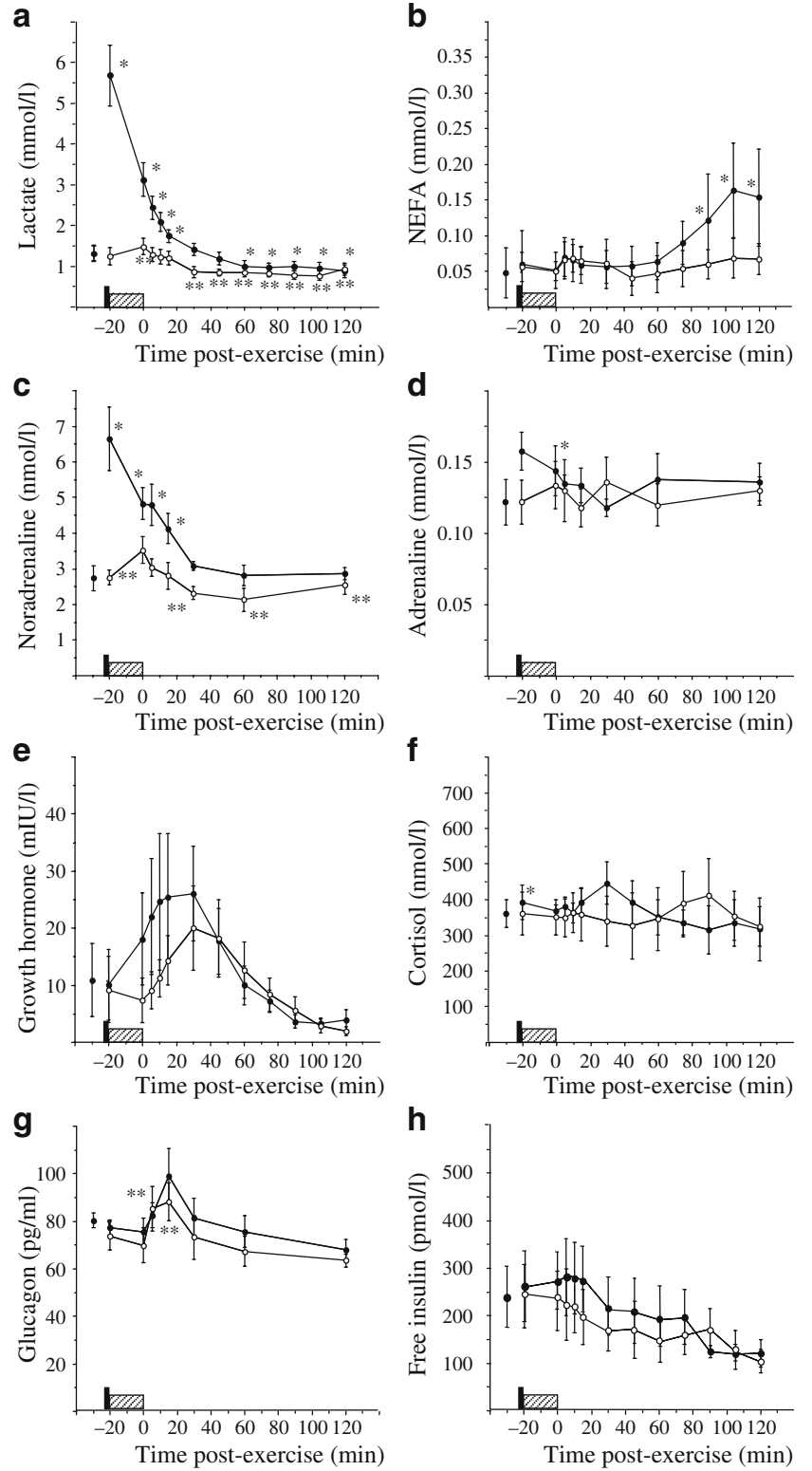

Fig. 2 Effect of a 10-s sprint on the levels of lactate (a), NEFA (b), noradrenaline (c), adrenaline (d), growth hormone (e), cortisol (f), glucagon (g) and free insulin (h) during and after moderate-intensity exercise. All values are shown as mean \pm SEM. Black boxes, sprint; hatched boxes, moderate-intensity exercise; closed circles, SP trial; open circles, CON trial. ${ }^{*} p<0.05$ compared with rest in SP; $* *<<0.05$ compared with rest in $\mathrm{CON}$

might have been masked by the large inter-individual variability in growth hormone levels. Nevertheless, even if growth hormone levels had increased significantly, this would probably have been of lesser importance because it has been reported that (1) administration of a growth hormone pulse does not affect peripheral glucose uptake in insulin-treated individuals with type 1 diabetes [9] and (2) the infusion of octreotide, an inhibitor of growth hormone release, has no acute effects on the magnitude of the hyperglycaemic effect of high-intensity exercise [10]. 
There is evidence that the aforementioned factors proposed to explain the stabilising effects of a short sprint on postexercise glycaemia might be similar, irrespective of whether sprinting is performed before, during or after moderateintensity exercise. Indeed, elevated lactate and catecholamine levels have also been proposed to stabilise glycaemia at the onset of recovery from moderate-intensity exercise interspersed either with several short sprints [4] or followed by a 10 -s sprint [2]. It is less clear, however, whether growth hormones are also involved under these conditions because of a lack of consistencies across studies. Clearly, more research is required to identify the factors responsible for the sprint-mediated stabilisation of glycaemia post-exercise.

On practical and clinical grounds, our findings suggest that before engaging in moderate-intensity exercise, individuals with type 1 diabetes should incorporate into their warm-up routine a short $10 \mathrm{~s}$ maximal sprint effort, especially as a 10 -s sprint is well tolerated [2]. However, before recommending the use of sprinting to prevent an early post-exercise fall in glycaemia, a number of issues must be addressed such as the extent to which the efficacy of prior sprinting is affected by the intensity and duration of the subsequent bout of exercise and the identification of any subpopulations of type 1 diabetic individuals unlikely to benefit from sprinting.

In conclusion, our findings show that sprinting before a bout of exercise of moderate intensity could be a novel tool of clinical importance for preventing the fall in glycaemia during early recovery in young healthy individuals with type 1 diabetes. These findings provide one of the very few examples [2-4] of exercise offering an immediate shortterm clinical benefit (delayed fall in glycaemia).

Acknowledgements This research was funded jointly by a Juvenile Diabetes Research Foundation (JDRF)/National Health Medical Research Council of Australia program grant to T. W. Jones and P. A.
Fournier. L. D. Ferreira is supported by a JDRF Fellowship. The authors acknowledge the technical assistance of L. Youngs and A. Thompson.

Duality of interest The authors declare that there is no duality of interest associated with this study.

\section{References}

1. Marliss EB, Vranic M (2002) Intense exercise has unique effects on both insulin release and its roles in glucoregulation: implications for diabetes. Diabetes 51:S271-S283

2. Bussau VA, Ferreira LD, Jones TW, Fournier PA (2006) The 10-s maximal sprint: a novel approach to counter an exercise-mediated fall in glycemia in individuals with type 1 diabetes. Diabetes Care 29:601-606

3. Guelfi KJ, Jones TW, Fournier PA (2005) Intermittent high-intensity exercise does not increase the risk of early postexercise hypoglycemia in individuals with type 1 diabetes. Diabetes Care 28:416-418

4. Guelfi KJ, Jones TW, Fournier PA (2005) The decline in blood glucose levels is less with intermittent high-intensity compared with moderate exercise in individuals with type 1 diabetes. Diabetes Care 28:1289-1294

5. Fairchild TJ, Fletcher S, Steele P, Goodman C, Dawson B, Fournier PA (2002) Rapid carbohydrate loading after a short bout of near maximal-intensity exercise. Med Sci Sports Exerc 34:980-986

6. Galassetti P, Tate D, Neill RA, Morrey S, Wasserman DH, Davis SN (2003) Effect of antecedent hypoglycemia on counterregulatory responses to subsequent euglycemic exercise in type 1 diabetes. Diabetes 52:1761-1769

7. Coker RH, Kjaer M (2005) Glucoregulation during exercise: the role of the neuroendocrine system. Sports Med 35:575-583

8. Vettor R, Lombardi AM, Fabris R et al (1997) Lactate infusion in anesthetized rats produces insulin resistance in heart and skeletal muscles. Metabolism 46:684-690

9. Møller N, Schmitz O, Møller J, Butler PC (1992) Effects of a physiological growth hormone pulse on substrate metabolism in insulin-dependent (type 1) diabetic subjects. J Clin Endocrinol Metab 75:432-436

10. Sigal RJ, Fisher S, Halter JB, Vranic M, Marliss EB (1996) The roles of catecholamines in glucoregulation in intense exercise as defined by the islet cell clamp technique. Diabetes 45:148-156 